\title{
Supra- und diakondyläre Frakturen des distalen Humerus
}

\author{
Kay Schmidt-Horlohé, Lars Becker, Reinhard Hoffmann
}

\section{Zusammenfassung}

Frakturen des distalen Humerus im Erwachsenenalter stellen eine seltene Verletzung dar. Die konservative Therapie durch Immobilisation führt fast immer zu erheblichen posttraumatischen Bewegungseinschränkungen. Das Ziel einer dauerhaft stabilen und schmerzfreien Gelenkfunktion ist in der Regel nur durch eine offene Reposition und übungsstabile Osteosynthese zu erreichen. Die operative Therapie wird durch eine komplexe Gelenkgeometrie, multifragmentäre Frakturformen und die oftmals schlechte Knochenqualität der im höheren Lebensalter zunehmend vorkommenden Fraktur erschwert. Um optimale Behandlungsergebnisse zu erzielen, sind detaillierte Kenntnisse der Anatomie und Biomechanik, der Zugangswege und geeigneten Implantate als auch der operativen Strategie erforderlich.

\section{Supra- and Diacondylar Fractures of the Distal Humerus}

Fractures of the distal humerus count for only a small number of all fractures in mature patients. As conservative treatment is frequently associated with a limited and painful elbow function, open reduction and stable internal fixation with early range of motion exercises are established as the gold standard. However, multifragmentary fractures with diminished bone quality in the growing elderly population represent a challenge for surgical treatment. Detailed knowledge of the complex articular anatomy, the biomechanics, operative approaches and available implants as well as an injury-specific treatment algorithm are needed for the successful management of these complex fractures.

\section{Anatomie und Biomechanik}

Das Ellenbogengelenk stellt eine aus 3 Gelenken bestehende funktionelle Einheit dar. Es besteht aus dem humeroulnaren, dem humeroradialen und dem proximalen radioulnaren Gelenk.

Der distale Humerus wird in einen ulnaren und radialen Pfeiler unterteilt. Diese enden gelenknah in den Epikondylen und tragen den artikulären Block. Dieser besteht im anterioren Anteil des distalen radialen Pfeilers aus dem Capitulum humeri, dem Gelenkpartner des Caput radii, während die am distalen ulnaren Pfeiler befindliche Trochlea humeri mit dem Olekranon artikuliert.

OP-JOURNAL 2012; 28: 4-12

(C) Georg Thieme Verlag KG Stuttgart · New York DOI http://dx.doi.org/10.1055/s-0031-1298550

Der distale Humerus stellt ein 3-dimensional hochkomplexes Gelenk dar.

In Bezug zur zentralen Humerusachse ist die Gelenkachse des distalen Humerus $4-8^{\circ}$ valgus anguliert, $3-4^{\circ}$ innenrotiert und in der Sagittalebene $30-40^{\circ}$ anterior gekippt (Abb. 1).

Weite Bereiche des distalen Humerus stellen gelenkbildende Strukturen dar, sodass lediglich wenige Lokalisationen einer Osteosynthese zur Verfügung stehen. Implantate können dorsoradial, medial und lateral positioniert werden. Um die Extension nicht zu blockieren, muss die Einengung der Fossa olecrani durch Implantatmaterial vermieden werden. Ebenso können von dorsal eingebrachte, in die ventral gelegene Fossa coronoidea oder radialis überragende Schrauben bei Beugung zu einem Impingement am Proc. coronoideus oder Radiuskopf führen.
Am Ellenbogen besteht eine enge anatomische Beziehung zu den Nerven und Gefäßen des Armes:

Der N. ulnaris durchdringt ca. $8 \mathrm{~cm}$ proximal des Epicondylus medialis das Septum intermusculare brachii mediale und verläuft dann am medialen Rand des Caput mediale des M. triceps nach distal. Er wird in diesem Bereich von der A. collateralis ulnaris posterior begleitet. Am dorsalen Aspekt des Epicondylus medialis zieht der N. ulnaris in den Sulcus N. ulnaris und tritt dann in die OsbornArkade zwischen den beiden Köpfen des Flexor carpi ulnaris ein.

10-12 cm proximal des Epicondylus lateralis tritt der N. radialis von posterior durch das Septum intermusculare brachii laterale und verläuft dann im Radialistunnel zwischen dem M. brachioradialis und brachialis nach anterior in die Ellenbeuge, wo er sich in einen Ramus profundus und superficialis aufteilt.

Distal der Medianusgabel verläuft der N. medianus, die A. brachialis kreuzend, im Sulcus bicipitalis medialis zur medialen Ellenbeuge und gelangt medial der Bizepssehne und der A. brachialis unter dem Lacertus fibrosus zwischen den beiden Köpfen des M. pronator teres zum Unterarm.

Die A. brachialis gelangt anterior des M. brachialis und lateral des N. medianus liegend zur Ellenbeuge. In Höhe des Radiuskopfs teilt sie sich in ihre Endäste, die A. radialis et ulnaris [12].

Abhängig von der Beuge- und Rotationsstellung des Unterarms werden eingeleitete Kräfte zu 60\% über die radiale und zu $40 \%$ über die ulnare Säule auf den Oberarm übertragen [10].

\section{Epidemiologie und Unfallmechanismus}

Distale Humerusfrakturen des Erwachsenen sind mit einer Inzidenz von ca. 


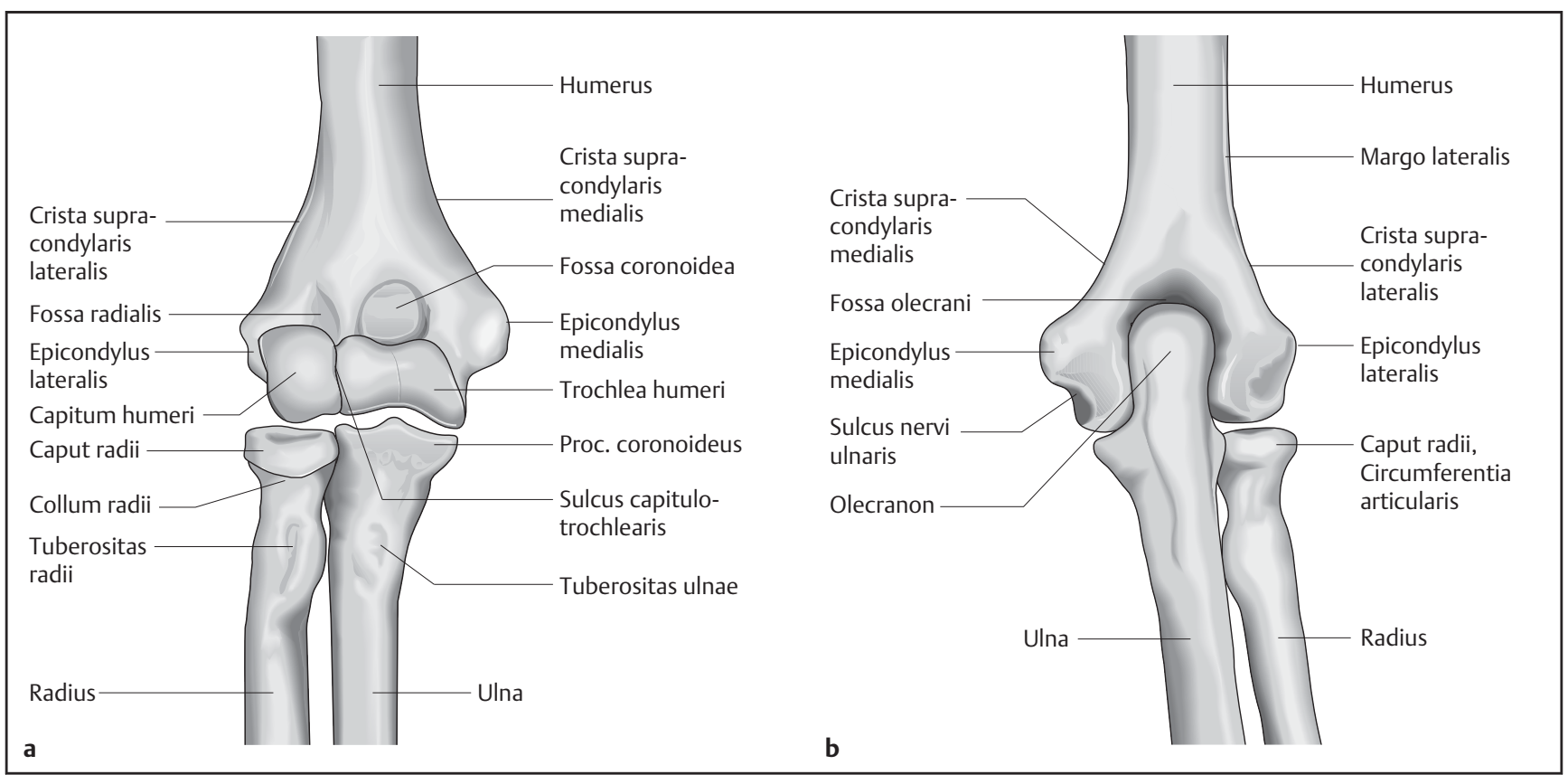

Abb. 1 a und $\mathbf{b}$ Knöcherne Anatomie des Ellenbogengelenks. a Ansicht von ventral, b Ansicht von dorsal.

5/100000 Einwohnern und einem Anteil von 2-6\% aller Frakturen eine relativ seltene Entität. Sie stellt aber mit ca. 30\% einen relevanten Anteil der knöchernen Verletzungen des Ellenbogengelenks dar.

Es findet sich eine typische bimodale Altersverteilung mit einem Gipfel bei jungen männlichen Patienten (oft hochenergetische Traumata) und einem weiteren Gipfel bei älteren Patientinnen über 65 Jahren (oft niedrigenergetisch).

Bei entsprechender Unfallanamnese sind Frakturen im Alter als osteoporotische Frakturen zu betrachten und sollten den Behandelnden zu einer entsprechenden Diagnostik und ggf. auch Therapie veranlassen.

Typischerweise findet sich bei älteren Patienten eine Kombination aus hochkomplexen Mehrfragmentfrakturen und schlechter Knochenqualität.

Bei jungen Patienten führen oftmals hochenergetische Mechanismen zur Fraktur, was insbesondere bei diesem Kollektiv in einer bis zu 30\% hohen Inzidenz von offenen Verletzungen resultiert. Bei älteren Patienten liegen oftmals einfache Stolperstürze vor, wobei auch hier durch eine durch Komorbiditäten geschädigte Haut (Kortisoneinnahme) ein sorgfältiges Weichgewebsmanagement angezeigt ist.
Die distale Humerusfraktur kann durch einen direkte (z.B. Pkw-Seitaufprall) oder indirekte Krafteinleitung entstehen. Neben der individuellen Knochenstruktur kommt der Ellenbogengelenkstellung während der Krafteinleitung eine wesentliche Bedeutung zu. So finden sich bei Sturz auf den extendierten Arm überwiegend suprakondyläre, auf den flektierten Arm überwiegend perkondyläre und interkondyläre Frakturformen. Sagittale Frakturmuster (Capitulum- und Trochleafrakturen) finden sich oftmals im Rahmen von Luxationsereignissen und sind entsprechend des Unfallmechanismus häufig mit begleitenden ligamentären Verletzungen vergesellschaftet.

Entsprechend der demografischen Entwicklung ist mit einer Zunahme der Inzidenz bei den distalen Humerusfrakturen im höheren Lebensalter auszugehen.

\section{Diagnostik}

Neben den unsicheren Frakturzeichen (Schmerz, Schwellung, Hämatom) findet sich üblicherweise eine schmerzhaft eingeschränkte Gelenkfunktion (functio laesa) und insbesondere bei den höhergradigen Frakturformen oftmals eine teils groteske Fehlstellung im Ellenbogengelenk.

Neben der Unfallanamnese kommt der allgemeinen Krankheitsanamnese internistischer Grunderkrankungen (Malig- nome, systemische Kortisontherapie) als auch bereits prätraumatischen bestehenden Gelenkstörungen (rheumatoide Arthritis, vorbestehende Bewegungslimitierung) eine besondere Bedeutung hinsichtlich der weiteren Therapieplanung zu.

Neben der Inspektion, Palpation und Bewegungsprüfung des Ellenbogengelenks ist die Untersuchung der angrenzenden Gelenke immer durchzuführen. Insbesondere bei axialen Stauchungstraumen sind Verletzungen im Bereich der Handwurzel und/oder des Handgelenks nicht unüblich. Die erhobenen Befunde müssen sorgfältigst dokumentiert werden, bei Weichgewebsschäden ist zur Verlaufsbeurteilung eine Wunddokumentation dringend $\mathrm{zu}$ empfehlen (Fotodokumentation).

Neben der konventionellen Röntgendiagnostik in 2 Ebenen ist bei komplexen Frakturen die Indikation zur CT-Diagnostik großzügig zu stellen.

Dreidimensionale Rekonstruktionen der Fraktur, ggf. mit Subtraktion des Unterarms, erleichtern das Frakturverständis maßgeblich und ermöglichen eine detaillierte OP-Planung. Bei Verdacht auf begleitende Gefäßläsionen ist eine Angio-CT oder Angiografie angezeigt.

Die MRT-Untersuchung hat außer bei Verdacht auf osteochondrale Läsionen 


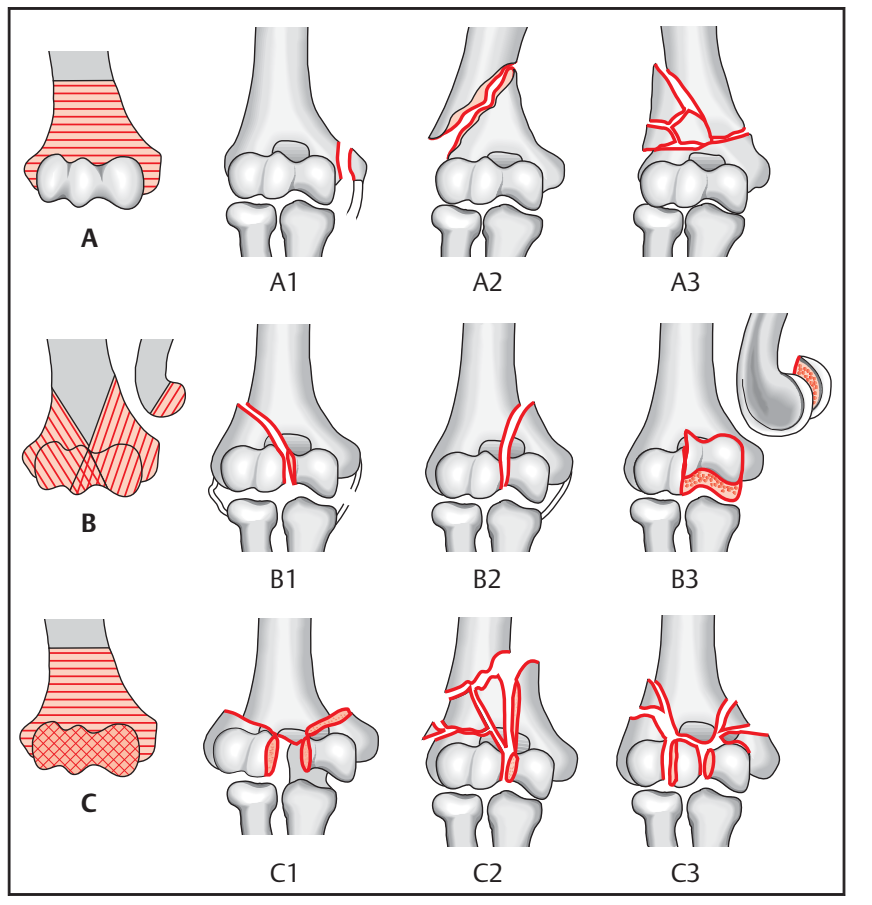

Abb. 2 Klassifikation distaler Humerusfrakturen entsprechend der $\mathrm{Ar}$ beitsgemeinschaft für Osteosynthesefragen.

keinen Stellenwert in der Akutphasediagnostik distaler Humerusfrakturen.

\section{Frakturklassifikation}

Frakturen des distalen Humerus sollten entsprechend der von der Arbeitsgemeinschaft für Osteosynthesefragen vorgeschlagenen AO-Klassifikation eingeteilt werden [5]. Andere, überwiegend im angloamerikanischen Raum angewandte Klassifikationen sind die Klassifikationen nach Mehne und Matta sowie Riseborough und Radin.

Bei der AO-Klassifikation werden vollständig extraartikuläre Frakturen (AFrakturen) von partiell intraartikulären Frakturen (B-Frakturen) und vollständig intraartikulären Frakturen (C-Frakturen) unterschieden.

Abhängig von der metaphysären und intraartikulären Verletzungsschwere erfolgt die Klassifikation in Subgruppen (Abb. 2). Die AO-Klassifikation ist sowohl von therapeutischer als auch von prognostischer Bedeutung.

Entsprechend einer epidemiologischen Studie aus England stellen A-Frakturen einen Anteil von 38\%, Typ-B-Frakturen einen Anteil von 24\% und Typ-C-Frakturen einen Anteil von 37\% dar. In derselben Untersuchung ist eindrücklich die Häufigkeit eines offenen Weichgewebsschadens in Abhängigkeit von der Frakund der Trochlea. Oestern klassifiziert.

\section{Therapieformen}

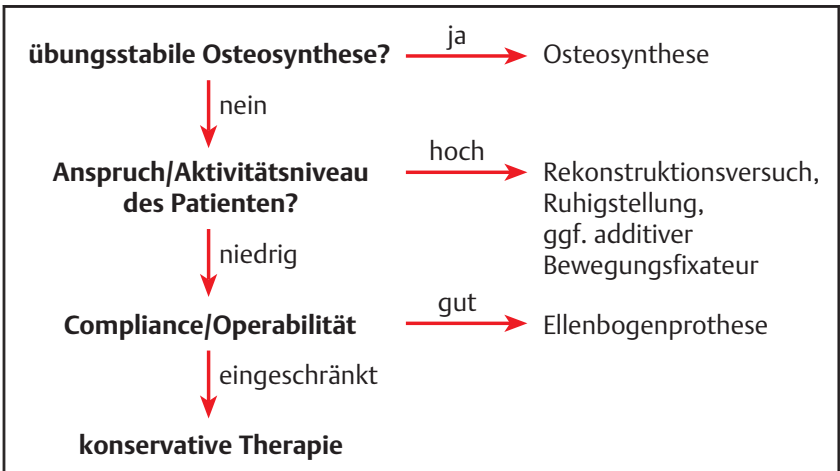

Abb. 3 Entscheidungsalgoritmus zur Behandlung distaler Humerusfrakturen an der Berufsgenossenschaftlichen Unfallklinik Frankfurt am Main.

Die Gefahr einer verzögerten oder gar ausbleibenden Bruchheilung ist um bis zum 6-Fachen erhöht, die funktionellen Ergebnisse sind überwiegend schlecht. Eine längere Ruhigstellung des Ellenbogengelenks führt besonders beim alten Patienten durch posttraumatische Gelenkkapselkontraktur, Narbenbildung und Ossifikationen nahezu unausweichlich zu einer signifikanten Einsteifung des Gelenks. Eine Ruhigstellung über 14 Tage sollte dringend vermieden werden. Die konservative Therapie ist somit nur indiziert, wenn ein operatives Verfahren wegen Begleiterkrankungen nicht möglich oder der Anspruch und die Compliance sehr niedrig ist (z.B. bei demenzieller Erkrankung, gebrauchsunfähige Extremität nach Schlaganfall). Im Fall einer konservativen Therapie ist nach initialer Schienenruhigstellung nach Abschwellung ein zirkulärer Oberarmgips mit Handeinschluss indiziert. Die vulnerable Weichgewebssituation ist bei multimorbiden Patienten (Kortisonhaut) stets kritisch zu bedenken.

Ziel der operativen Therapie distaler Humerusfrakturen ist die Wiederherstellung einer dauerhaft stabilen und schmerzfreien Gelenkfunktion.

mehrfragmentäre Capitulumfrakturen. Vervollständigt wird die Klassifikation durch den von McKee ergänzten Typ IV, einer Abscherfraktur des Capitulums

Weichgewebsverletzungen werden nach Gustilo und Anderson oder Tscherne und

Die bis Mitte des letzten Jahrhunderts durchgeführte konservative Therapie distaler Humerusfrakturen führte in einem hohen Prozentsatz zu unbefriedigenden Ergebnissen.

Diverse klinische Studien konnten nachweisen, dass dies nur durch eine exakte anatomische Rekonstruktion der Gelenkflächen und Gelenkgeometrie sowie eine frühfunktionelle Nachbehandlung möglich ist $[2,4,7]$. Die offene Reposition und stabile interne Fixation gilt somit als Therapie der Wahl. Ist eine primäre Übungsstabilität allein durch die Osteosynthese nicht zu erreichen, sollte über die additive Stabilisierung durch einen Fixateur oder Bewegungsfixateur nachgedacht werden. Obwohl hinsichtlich der späteren Gelenkbeweglichkeit die frühfunktionelle Behandlung von enor- 


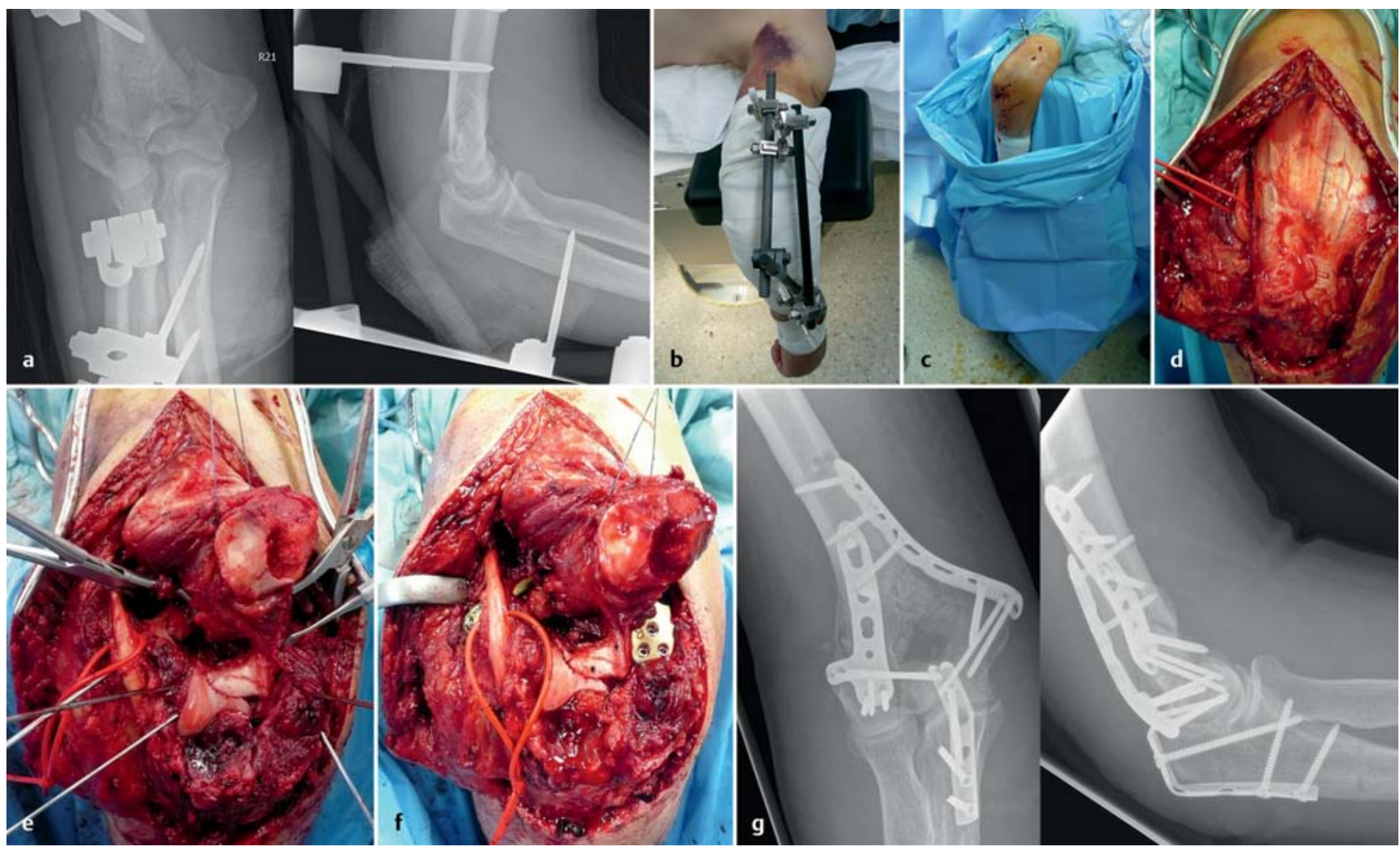

Abb. 4 a bis $\mathbf{g}$ Komplexe intraartikuläre distale Humerusfraktur (AO 13 C3), männlich, 56 Jahre. a Frakturröntgen nach Fixateur-externe-Stabilisierung, b Lagerung des Patienten in Bauchlage, c sterile Abdeckung des OP-Bereichs, $\mathbf{d}$ dorsaler Zugang und Darstellung des N. ulnaris, e Darstellung der Fraktur über Olekranonosteotomie, temporäre K-Draht-Stabilisierung nach anatomischer Reposition, $\mathbf{f}$ Doppelplattenosteosynthese am dorsoradialen und medialen Pfeiler, $\mathbf{g}$ postoperative Röntgenkontrolle.

mer Wichtigkeit ist, hat dennoch die knöcherne Heilung einen höheren Stellenwert. Die Erfolgsaussichten sind nach einer sekundären Arthrolyse bei Steife besser als nach knöchernen Heilungsstörungen. Somit ist bei nicht ausreichender Stabilität dennoch eine Ruhigstellung angezeigt.

Im Falle von erheblicher intraartikulärer Zerstörung, vorbestehenden Gelenkpathologien und höherem Lebensalter ist der endoprothetische Gelenkersatz zu erwägen (siehe Artikel Dr. Becker et al. in dieser Ausgabe, S. 14).

Die primäre Arthrodese des Ellenbogengelenks in Funktionsstellung ist selten und kommt nur bei ausgewählten $\mathrm{Pa}-$ tienten in Betracht.

Abb. 3 beschreibt den in der Berufsgenossenschaftlichen Unfallklinik Frankfurt am Main etablierten Entscheidungsalgorithmus hinsichtlich der Therapieform (Abb. 3).

\section{OP-Vorbereitung, Lagerung und operative Zugänge}

Der Operationszeitpunkt hängt im Wesentlichen vom begleitenden Weichgewebstrauma (offene Fraktur), Begleitverletzungen (Gefäßläsionen) und logistischen Komponenten ab.

Insbesondere bei komplexen Typ-CFrakturen ist ein erfahrenes OP-Team notwendig. Da eine Notfallindikation selten vorliegt, ist eine operative Versorgung der Fraktur im Intervall bei optimalen Grundvoraussetzungen gerechtfertigt. Bei eventueller Notwendigkeit eines endoprothetischen Gelenkersatzes ist ein entsprechendes Instrumentarium zur OP vorzuhalten.

Ist eine zeitnahe Versorgung der Fraktur nicht gewährleistet, muss natürlich temporär eine Gipsschienenruhigstellung oder, in Abhängigkeit von Begleitverletzungen und Weichgewebsschaden, die Anlage eines gelenküberbrückenden Fixateur externe erfolgen.

Um eine schmerzfreie postoperative Nachbehandlung zu ermöglichen, ist die
Anlage eines Schmerzkatheters zu erwägen. Nachteilig ist hierbei allerdings die unter Umständen postoperative eingeschränkte Beurteilbarkeit der Motorik und Sensibilität. Ob ein Schmerzkatheter das funktionelle Ergebnis positiv beeinflusst, ist nicht wissenschaftlich belegt.

Ein Blasendauerkatheter sollte bei zu erwartender OP-Zeit von über 2 Stunden angelegt werden.

Die perioperative Antibiotikaprophylaxe sollte 30 Minuten vor Hautschnitt und ggf. Schließen der Oberarmblutsperre erfolgen.

Die Oberarmblutsperre sollte immer vorbereitet werden, ob diese dann genutzt wird, hängt vom OP-Verlauf ab. Eine Blutsperre über 120 Minuten sollte vermieden werden. Wichtig ist, dass die Blutsperre weit proximal angelegt wird, um ggf. eine Erweiterung des Zugangs nach proximal nicht zu behindern.

Der Patient wird entsprechend des nach der Fraktursituation gewählten Zugangs gelagert. Im Falle eines dorsalen Zugangs empfiehlt sich die Lagerung in Bauchla- 
ge, die verletzte Extremität wird dabei über einen kurzen Armtisch oder eine Rolle ausgelagert. Eine Beugung bis mindestens $100^{\circ}$ muss gewährleistet sein. Alternativ ist der dorsale Zugang auch in Seitenlage oder Rückenlage durchführbar. Bei Bauch- oder Seitenlage hat es sich im eigenen Vorgehen bewährt, den Arm frei in einem Tischabdecktuch hängen zu lassen. Die durch die große Wundfläche doch erhebliche Blutung wird so aufgefangen und der Arm ist stets steril umgeben (Abb.4). In Rückenlage empfiehlt es sich, den Arm auf einer Beinschale über dem Oberkörper des Patienten zu lagern.

Im Falle eines medialen, lateralen oder ventralen Zugangs wird der Patient in Rückenlage mit auf dem Armtisch ausgelagertem Arm gelagert.

Bei im CT zu erahnender artikulärer oder metaphysärer Trümmerfraktur mit Substanzverlust ist die Indikation zur Spongiosaplastik großzügig zu stellen. In diesem Fall muss eine Entnahmestelle (z.B. am vorderen oder hinteren Beckenkamm) zugänglich sein.

Welcher operative Zugang gewählt wird, hängt maßgeblich von der Frakturmorphologie und den Begleitverletzungen ab.

\section{Radialer Zugang/proximal limitierter Zugang nach Kocher}

Indikation: unikondyläre Frakturen des radialen Pfeilers, Capitulumfrakturen

Die Hautinzision beginnt ca. $5 \mathrm{~cm}$ proximal des Ellenbogengelenks und zieht über den radialen Epikondylus nach distal, posterior des Radiusköpfchens. Je nach Höhe der radialen Pfeilerfraktur ist ggf eine Erweiterung nach proximal notwendig. Nach Durchtrennung der Faszie wird proximal das Intervall zwischen dem posterior gelegenen $M$. triceps und dem brachioradialis dargestellt, nach distal verläuft anterior der M. extensor carpi ulnaris und posterior der M. anconeus. Nach Darstellung der oftmals bereits traumatisch eröffneten Gelenkkapsel ist der radiale Gelenkanteil einsehbar. Ein subperiostales Präparieren nach anterior und posterior ermöglich die Visualisierung des gesamten radialen Pfeilers. Ventral des Zugangs ist auf den dort verlaufenden N. radialis zu achten. Ein übermäßiger Zug mit Retraktoren und Haken muss vermieden werden.

\section{Ulnarer Zugang}

Indikation: unikondyläre Frakturen des ulnaren Pfeilers, Trochleafrakturen

Die Hautinzision beginnt medial, ebenfalls $5 \mathrm{~cm}$ proximal des Gelenks, und setzt sich über den medialen Epikondylus nach distal fort. Das Aufsuchen des $\mathrm{N}$. ulnaris ist proximal am medialen Rand des M. triceps am leichtesten. Nach Darstellung des N. ulnaris wird dieser dann sparsam neurolysiert und mittels Vessel Loop angeschlungen. Nach subperiostalem Ablösen der Flexorenmuskulatur wird die Gelenkkapsel eröffnet. In der Modifikation dieses Zugangs nach Campbell wird der Epicondylus medialis osteotomiert und mit den Muskelansätzen nach ventral verlagert.

\section{Ventraler Zugang}

Indikation: Capitulum- und Trochleafrakturen

Beim ventralen Zugang nach Henry erfolgt eine S-förmige Hautinzision von proximal-lateral über die Fossa cubitalis nach distal-medial. Der im Zugangsbereich verlaufende $\mathrm{N}$. cutaneus antebrachii lateralis sollte geschont werden, da ansonsten eine Dys- oder Anästhesie der sensibel versorgten Hautareale resultieren kann. Die sensibel innervierten Hautareale erstrecken sich am dorsalen und volaren radialseitigen Unterarm. Volar ist sein Versorgungsgebiet größer und reicht bis zum Daumenballen, dorsal endet es etwa in Höhe des Handgelenks. Nach Eröffnung der Unterarmfaszie wird das Muskelintervall zwischen M. brachialis und brachioradialis aufgesucht. N. medianus und A. brachialis werden nach medial, der $\mathrm{N}$. radialis nach lateral gehalten. Nach Inzision der ventralen Gelenkkapsel sind das Capitulum und der radiale Anteil der Trochlea darstellbar.

\section{Dorsale Zugänge}

Indikation: uni- und bikondyläre Frakturen des medialen und/oder lateralen Pfeilers

Der dorsale Zugang zum Ellenbogengelenk stellt den Standardzugang in der Versorgung komplexer Frakturen dar.

Je nach artikulärer Verletzungsschwere kann über die im Folgenden dargestellten Zugänge ein größtmöglicher Anteil der Gelenkfläche eingesehen werden.
Insbesondere Begleitverletzungen des proximalen Unterarms können über diesen Zugang leicht mitbehandelt werden. Des Weiteren ergeben sich Vorteile durch eine geringere Hautnervendichte und ein besseres kosmetisches Ergebnis, da eine dorsal am Ellenbogengelenk lokalisierte Narbe im Alltag kaum auffällt. Zudem ist die Verbandspflege bei ggf. additivem Fixateur externe einfacher möglich.

Die dorsale Hautinzision erfolgt unter Radialumschneidung des Olekranons und erstreckt sich je ca. $10 \mathrm{~cm}$ nach proximal und distal. Bei traumatischer Bursaverletzung ist eine Bursektomie $\mathrm{zu}$ empfehlen. Nach Darstellung des M. triceps muss obligat das Identifizieren und Markieren des N. ulnaris im Sulcus N. ulnaris erfolgen. Eine extraanatomische Transposition des Nervs nach ventral wird im eigenen Vorgehen nur durchgeführt, wenn nach Frakturstabilisierung eine mechanische Irritation durch die Osteosynthese befürchtet werden muss. Aktuelle Studien konnten keinen Nutzen der Transposition nach ventral belegen. Der Anteil an Nervenirritationen ist nach ventraler Transposition allerdings bis zu 4-fach erhöht. Von immenser Wichtigkeit, insbesondere im Hinblick auf eine spätere Revision oder Metallentfernung, ist die genaue Dokumentation der Nervenposition in der OP-Info und des OP-Berichts. Bei der Präparation nach proximal am radialen Pfeiler ist auf den im distalen Drittel des Humerusschafts von medioproximal nach lateroventral kreuzenden $\mathrm{N}$. radialis $\mathrm{zu}$ achten.

Abhängig von der Frakturmorphologie ermöglicht die dorsale Hautinzision verschiedene Zugangswege.

\section{Zugang nach Alonso-Llames (triceps-on)}

Durch Präparation eines medialen und lateralen Fensters am Trizeps kann eine gute Darstellung des metaphysären distalen Humerus erreicht werden. Die Gelenkfläche ist nur begrenzt einsehbar. Dieser Zugang ist daher in erster Linie für extraartikuläre und einfach intraartikuläre Frakturen geeignet. Vorteilhaft ist, dass die einsehbare artikuläre Fläche des distalen Humerus leicht durch eine Erweiterung dieses Zugangs im Sinne einer Olekranonosteotomie vergrößert werden kann. Des Weiteren ermöglicht dieser Zugang ein Umschwenken auf den unten beschriebenen Bryan-Morrey-Zugang (bei Entscheidung zur Endoprothe- 
se). Daher ist der Alonso-Llames-Zugang auch zur Erstbeurteilung komplexer Frakturen gut geeignet, da er abhängig von der Verletzungsschwere erweitert werden kann.

\section{Transolekranärer Zugang}

Dieser wurde erstmals 1915 von MacAusland beschrieben und in der Folge wiederholt modifiziert. Eine V-förmige Osteotomie des Olekranons ist durch die höhere Rotationsstabilität und größere knöcherne Kontaktfläche einer geraden Osteotomie vorzuziehen. Entsprechend der Form der Osteotomie wird diese auch als Chevron-Osteotomie bezeichnet. Chevron ist der englische Begriff für Winkel und beschreibt in der Architektur das V-förmige Aufeinandertreffen von Dachbalken. Es wurde als Zeichen eines Verdiensts im Militär eingeführt und dient heute in erster Linie als Rangabzeichen.

Mit einer oszillierenden Säge werden die dorsalen $2 / 3$ des Olekranons osteotomiert, das letzte Drittel wird mit einem Lambotte-Meißel (benannt nach Albin Lambotte, belgischer Chirurg und Mitbegründer der Unfallchirurgie) frakturiert, um bei der Rekonstruktion eine gute Verzahnung der Fragmente zu erreichen. Das mobilisierte Olekranon wird dann in eine feuchte Kompresse eingeschlagen und kann so, mit anhaftendem Trizeps, nach proximal abgehoben werden (Abb. 4). Nach Vollendung der Osteosynthese erfolgt die Refixierung mittels Zuggurtung oder Plattenosteosynthese [13].

Der Anteil der nach Olekranonosteotomie einsehbaren Gelenkfläche ist im Vergleich zu anderen Zugängen um bis zu 50\% vergrößert.

\section{Trizeps-Splitting-Zugang}

Nach mittiger Durchtrennung des M. triceps wird der Streckapparat nach medial und lateral subperiostal am Olekranon und der proximalen Ulna unter Bildung von muskulofaszialen medialen und lateralen Lappen abgelöst.

\section{Bryan-Morrey-Zugang}

Ist die Implantation einer Ellenbogenprothese augrund der artikulären Verletzungsschwere wahrscheinlich, sollte die Gelenkdarstellung über einen den Streckmechanismus erhaltenden Zugang (z.B. Bryan-Morrey-Zugang) erfolgen, um die postoperative Rehabilitation unter

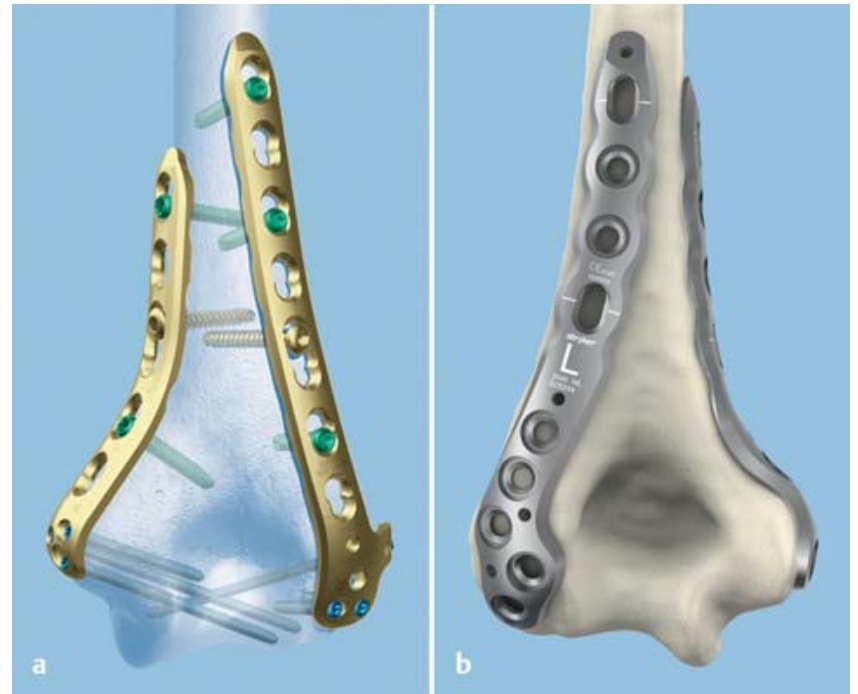

Abb. 5 a und $\mathbf{b}$

Winkelstabile, anatomisch präformierte Implantate für den distalen Humerus. a LCP Distal Humerus Plates System, Synthes, Umkirch. b Variax Elbow System, Stryker, Selzach, Schweiz.

aktiver Extension zu ermöglichen. Beim Zugang nach Bryan-Morrey wird der gesamte Streckapparat in seiner Kontinuität erhalten und von medial nach lateral subperiostal am Olekranon abgelöst.

\section{OP-Verfahren}

\section{Osteosynthesematerialien}

Fixateur externe

Bei frakturbedingt erheblicher Instabilität mit begleitendem, hochgradig offenem oder geschlossenem Weichgewebstrauma stellt die Ruhigstellung im Fixateur externe die primäre Stabilisierung der Wahl dar. Insbesondere im Rahmen einer Polytraumatisierung erlaubt der Fixateur externe im Gegensatz zur Gipsschienenruhigstellung eine vereinfachte Behandlungspflege.

Die Platzierung der Schanz-Schrauben erfolgt humeral und ulnar lateral.

Humeral ist die Platzierung der Pins über einen limitierten Zugang zur Schonung des im Übergang vom mittleren zum distalen Drittel nach lateral kreuzenden $\mathrm{N}$. radialis dringlichst zu empfehlen.

Bereits in dieser Phase sollte die Position der Pins mit Hinblick auf die definitive Versorgung gewählt werden. Die Pinlöcher stellen eine Schwächung der kortikalen Struktur dar und können bei an diesen Punkten endender Osteosynthese oder Prothese eine periimplantäre Fraktur begünstigen. Bei definitiver Prothesenversorgung ist zusätzlich das Risiko eines Zementaustritts gegeben.
Nach Stabilisierung des Allgemeinzustands des Patienten und Konsolidierung der Weichgewebe erfolgt dann in der Sekundärphase die definitive operative Versorgung der Fraktur.

Zusätzlich kann der Fixateur externe bei verbliebener Instabilität nach Osteosynthese Verwendung finden.

Kirschner-Drähte

Die perkutane Verschraubung und isolierte K-Draht-Osteosynthese bietet keine ausreichende Primärstabilität, um eine funktionelle Nachbehandlung $\mathrm{zu}$ gewährleisten und ist somit obsolet. KDrähte werden für die temporäre Fixierung benötigt (s.u.).

\section{Schrauben}

Es kommen Kleinfragmentschrauben und kanülierte Schraubensysteme zur Anwendung. Eine isolierte Schraubenosteosynthese bietet selten eine primäre Übungsstabilität, sodass eine Schraubenosteosynthese in der Regel durch eine Plattenosteosynthese gesichert wird.

Platten

Drittelrohrplatten besitzen keine ausreichende Stabilität, in der Vergangenheit wurden wiederholt Brüche dieser Platten beobachtet.

Es kommen daher LC-DC- oder 3,5-mmRekonstruktionsplatten zur Verwendung.

Die seit einigen Jahren angebotenen präformierten Osteosyntheseplatten erleich- 
tern das Anpassen an die komplexe Geometrie des distalen Humerus.

\section{Winkelstabile Platten}

Insbesondere bei komplexen Frakturen mit kleinen Fragmenten und schlechter Knochensubstanz bieten winkelstabile Osteosyntheseplatten relevante Vorteile.

Durch das Prinzip der Winkelstabilität kann oftmals eine gute Primärstabilität trotz o.g. Kombination erzielt werden. Durch die biomechanischen Eigenschaften der Plattenfixateure mit verbessertem Schraubenhalt sind im hohen Prozentsatz trotz schlechter Knochenqualität übungsstabile Frakturversorgungen zu erreichen $[3,14]$.

Dies gilt besonders für die neuen anatomisch vorgeformten und winkelstabilen Plattensysteme, die für den distalen Gelenkblock multiple Schraubenoptionen bereit halten $[9,11]$. Diese sind sowohl uni- als auch multidirektional winkelstabil verfügbar (Abb. 5). Entsprechend der Biomechanik der Winkelstabilität sollte die periostale Durchblutung durch das Fixateur-interne-Prinzip geschont werden. Da in biomechanischen und klinischen Studien wiederholt ein Versagen der am radialen Pfeiler von dorsal eingebrachten Schrauben beobachtet wurde, sollte eine lateromediale Verschraubung des Gelenkblocks stets angestrebt werden $[2,11]$.

Bei Hybridfixierung (artikulär winkelstabil/diaphysär konventionell) muss die Osteosyntheseplatte im Schaftbereich nachmodelliert werden, um eine sekundäre Verschiebung des Gelenkblocks zu verhindern (die Platte wird an den $\mathrm{Hu}$ merusschaft herangezogen). Werden die Platten als Fixateur interne verwendet (winkelstabile Fixierung artikulär und diaphysär), ist eine Anpassung in der Regel nicht notwendig (winkelstabile Fixierung ohne notwendigen Kontakt zwischen Platte und Knochenschaft).

Durch die neuen Implantatsysteme wurde die Möglichkeit einer gelenkerhaltenden Therapie auch hin zu hochkomplexen Frakturen mit begleitend schlechter Knochenqualität erweitert.

Die deutlich höheren Kosten für anatomisch präformierte, winkelstabile Implantate müssen allerdings unter wirtschaftlichen Gesichtspunkten kritisch betrachtet werden.
Bis dato fehlen klinisch vergleichende Studien zwischen konventionellen und winkelstabilen Implantaten, sodass lediglich eine Grad-I-Empfehlung zur Verwendung der winkelstabilen Implantate gegeben werden kann $[6,16]$.

\section{Frakturversorgung}

\section{A-Frakturen}

A1-Frakturen treten typischerweise bei noch nicht vollständig geschlossener Epiphysenfuge auf und stellen knöcherne Ausrisse des radialen oder ulnaren Kollateralbands dar. Nur bei diesen Frakturformen ist die isolierte Verschraubung mit Kleinfragment- oder kanülierten Schrauben gerechtfertigt.

Extraartikuläre, metaphysär einfache und mehrfragmentäre Frakturen werden nach offener Reposition mittels einer Doppelplattenosteosynthese am radialen und ulnaren Pfeiler stabilisiert. Die Lage der Platten kann radial und medial, alternativ aber auch dorsoradial und medial erfolgen. Die beidseitige Positionierung dorsal sollte biomechanischen Studien zufolge wegen der deutlich geringeren Primärsteifigkeiten vermieden werden. Der Gelenkblock sollte mit mindestens 4 Schrauben fixiert sein, am Schaft sind 6 bikortikale Schrauben gefordert. Winkelstabile Implantate sind bei guter Knochenqualität und großem Gelenkblock nicht notwendig, können aber bei schlechter Knochenqualität, metaphysärer Zertrümmerung und kleinem Gelenkblockfragment hilfreich sein.

\section{B-Frakturen}

Frakturen des radialen oder ulnaren Pfeilers werden entsprechend der Lokalisation durch eine einseitige Plattenosteosynthese stabilisiert. Eine isolierte interfragmentäre Verschraubung bietet keine ausreichende Primärstabilität und ist daher immer durch eine Plattenosteosynthese zu ergänzen.

Sagittale Abscherfrakturen können nach anatomischer Reposition direkt von ventral oder auch indirekt von dorsal verschraubt werden. Wichtig ist, dass keine Osteosynthesematerialien die Knorpeloperfläche überragen.

\section{C-Frakturen}

Es erfolgt zunächst die offene Rekonstruktion des Gelenkblocks. Nach Darstellung und Bergung der oftmals grob dislozierten artikulären Fragmente werden diese anatomisch reponiert und temporär fixiert. K-Drähte der Stärke $0,8-1,4 \mathrm{~mm}$ sind in diesem Schritt zur Stabilisierung ausreichend. Kleine osteochondrale Fragmente können ggf. mit resorbierbaren Pins fixiert werden.

Eine interfragmentäre Kleinfragmentschraube mit durchgehendem Gewinde oder eine kanülierte Schraube erhöhen die Stabilität des Gelenkblocks.

Im Anschluss wird der Gelenkblock mit K-Drähten der Stärke 1,6-2 mm gegen den Humerusschaft fixiert. In diesem Schritt muss die physiologische Angulation des Gelenkblocks im 30 ${ }^{\circ}$-Winkel nach anterior beachtet werden. Vor allem in Bauchlage und bei kräftigem umgebendem Weichgewebsmantel ist diese Beurteilung oftmals schwierig. Im Zweifelsfall sollte eine Durchleuchtungsuntersuchung erfolgen. Wie auch bei den A2- und A3-Frakturen ist die definitive Stabilisierung durch eine Doppelplattenosteosynthese $\mathrm{zu}$ realisieren. Die Lage der Platten kann analog radial und medial, alternativ aber auch dorsoradial und medial erfolgen. Es besteht anhaltend eine Kontroverse, ob die Platten in einer $90^{\circ}$-Konfiguration (dorsoradial und medial) oder $180^{\circ}$-Konfiguration (radial und medial) positioniert werden sollten. Biomechanische Untersuchungen mit simulierter metaphysärer Trümmerzone legen eine Positionierung in $180^{\circ}$ nahe. Allerdings konnte in der einzigen existierenden klinisch vergleichenden Studie zwischen $90^{\circ}$ - und $180^{\circ}$-Position kein Unterschied festgestellt werden. Fallserien zeigen sowohl in $90^{\circ}$ als auch in $180^{\circ}$ gleiche funktionelle Ergebnisse mit vergleichbaren Komplikationsraten.

Liegt eine metaphysäre Trümmerfraktur mit Defektsituation vor, ist die primäre Spongiosaplastik dringlichst zu empfehlen.

Alternativ ist eine primäre metaphysäre Verkürzung von einigen Zentimetern ohne funktionelle Einschränkungen der Streckmuskulatur möglich. Es ist dann allerdings von besonderer Wichtigkeit, die Fossa olecrani, coronoidea und radialis zur remodellieren (z.B. OuterbridgeKashiwagi Procedure).

Nach Abschluss der Osteosynthese und provisorischer Refixierung des Olekranons muss eine Bewegungsprüfung im vollen Bewegungsausmaß erfolgen. Eine 


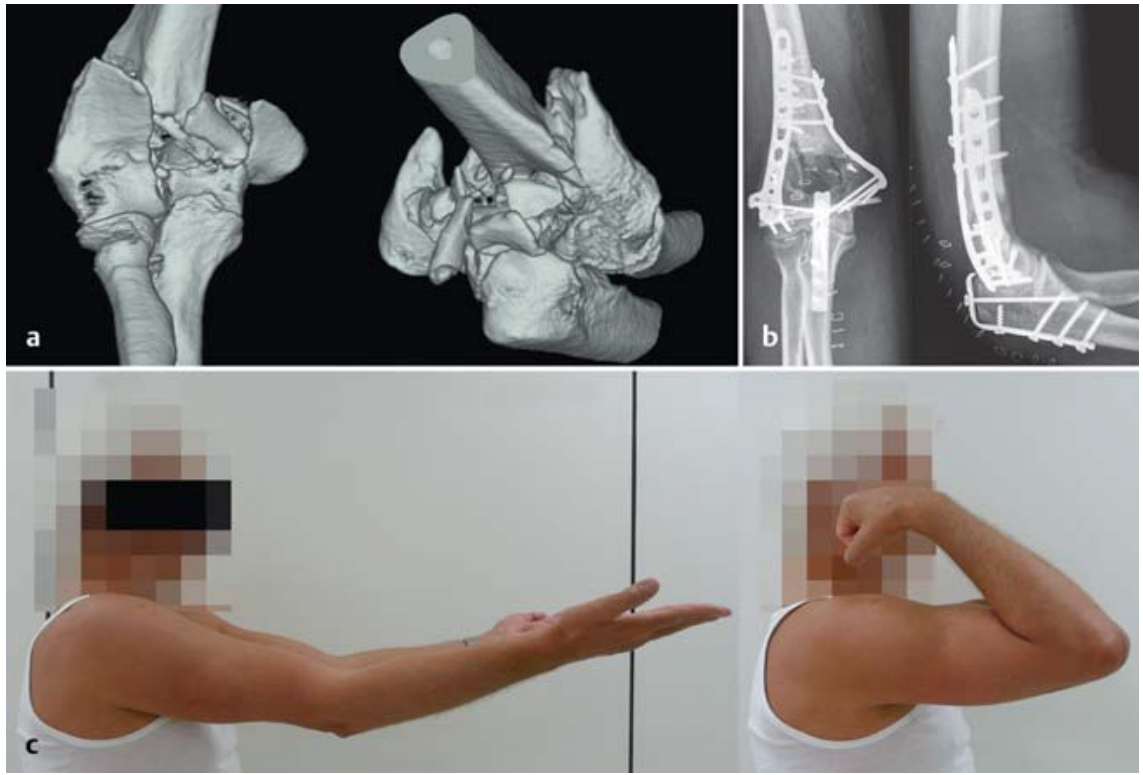

Abb.6a bis c AO-13-C3-Fraktur, männlich, 61 Jahre. a CT-Oberflächenrekonstruktion der Fraktur, b nach offener Reposition und winkelstabiler Doppelplattenosteosynthese, c funktionelles Ergebnis nach 20 Monaten entsprechend Mayo Elbow Performance Score sehr gut.

intraoperative Röntgenkontrolle zur Beurteilung der Reposition und Osteosynthese ist obligat durchzuführen.

Ist eine Osteosynthese technisch nicht möglich oder bestanden bereits prätraumatische, erhebliche Einschränkungen der Gelenkfunktion, ist der endoprothetische Ersatz zu erwägen (siehe Artikel Becker et al. in dieser Ausgabe).

Die Arthrodese des Ellenbogengelenks im Rahmen einer akuten distalen Humerusfraktur spielt eine untergeordnete Rolle.

\section{Nachbehandlung}

Ziel der operativen Therapie distaler Humerusfrakturen ist die Wiederherstellung einer dauerhaft schmerzfreien und stabilen Gelenkfunktion in einem für den Alltag ausreichenden Bewegungsausmaß. Dieses Ziel ist nur durch eine anatomische Rekonstruktion der Gelenkfläche und primär übungsstabile Osteosynthese zu erreichen.

Die frühe funktionelle Nachbehandlung hat einen signifikanten Einfluss auf das spätere Ergebnis [2,7].

Trotz initial stabiler Osteosynthese wird im eigenen Vorgehen postoperativ eine Gipsschiene angelegt. Dies unterstützt durch eine kurzzeitige (2-3 Tage) Ruhigstellung die Weichgewebskonsolidierung der initial oftmals erheblich geschwolle- nen Extremität, andererseits hat es nach unserer Erfahrung einen positiven Effekt hinsichtlich der postoperativen Schmerzen. Das funktionelle Ergebnis wird durch eine so kurze Ruhigstellung nicht beeinflusst. Lokale Kälteanwendungen und später Lymphdrainage unterstützen im Verlauf das Abschwellen.

Die Wirksamkeit einer heterotopen Ossifikationsprophylaxe nach Frakturen des Ellenbogengelenks ist wissenschaftlich nicht belegt, sollte aber dennoch prophylaktisch für einen Zeitraum von 4 Wochen mit einem nichtsteroidalen Antirheumatikum durchgeführt werden. Eine präoperative Bestrahlung bei der Primär-OP ist wegen des erhöhten Risikos einer Pseudarthrosenbildung nicht zu empfehlen. Im Rahmen von Revisionsoperationen und vorausgegangenen heterotopen Ossifikationen wird sie im eigenen Vorgehen aber auch angewendet.

Eine belastungsstabile knöcherne Konsolidierung tritt am distalen Humerus zwischen der 8. und 10. Woche ein. Eine Vollbelastung kann dann initiiert werden.

Die Indikation zur Entfernung des Osteosynthesematerials am distalen Humerus sollte wegen der durch Narbenbildung erhöhten Gefahr der iatrogenen Nervenverletzung sehr zurückhaltend gestellt werden.
Die Indikation sollte nur bei mechanischer Irritation durch das Implantatmaterial oder bei sensomotorischen Störungen gestellt werden. Eine umfangreiche Aufklärung des Patienten über Komplikationen und Risiken dieser vom Patienten oftmals als „kleine OP“ wahrgenommenen Eingriffs ist dringend $\mathrm{zu}$ empfehlen.

\section{Komplikationen}

Ein Osteosyntheseversagen, in der Regel durch Ausriss der Schrauben im Gelenkblock, sowie die Bildung einer Pseudarthrose wird in einem Prozentsatz von 7,5 bis $32 \%$ gesehen $[2,8,9,11,15]$.

Eine postoperative sensomotorische Störung des N. ulnaris, meist nur passager, ist eine der häufigsten Komplikationen nach operativer Therapie distaler Humerusfrakturen und wird mit einer Häufigkeit von bis zu $16 \%$ angegeben. Die anteriore Transposition des Nervs hat keinen protektiven Einfluss.

Ein Osteosyntheseversagen oder eine ausbleibende Konsolidierung nach Refixierung der Olekranonosteotomie findet sich in bis zu $31 \%$ der Fälle $[1,11]$.

\section{Funktionelle Ergebnisse}

Trotz der anhaltend hohen Komplikationsraten sind die Ergebnisse nach Osteosynthese distaler Humerusfrakturen in aktuellen Publikationen vielversprechend.

Wenn auch noch nicht durch vergleichende Studien belegt, könnte dies Ausdruck der durch neue, anatomisch präformierte und winkelstabile Implantate verbesserten Fragmentfixierung und somit frühen funktionellen Nachbehandlung sein. Gute und sehr gute Ergebnisse nach C3-Fraktur wurden bei über $90 \%$ der Patienten festgestellt (Abb. 6) $[9,11]$. Demgegenüber konnten nach konventioneller Plattenosteosynthese von C3Frakturen nur in 26\% der Fälle gute und sehr gute Resultate ermittelt werden [2].

\section{Literatur}

1 Gupta R. Intercondylar fractures of the distal humerus in adults. Injury 1996; 27 569-572

2 Korner J, Lill H, Muller LP et al. Distal humerus fractures in elderly patients: results after open reduction and internal fixation. Osteoporos Int 2005; 16 (Suppl. 2) S73-S79

3 Korner I, Lill H, Muller LP et al. The LCP-concept in the operative treatment of distal humerus fractures - biological, biomechanical 
and surgical aspects. Injury 2003; 34 (Suppl. 2) B20-B30

${ }^{4}$ Morrey BF. Current concepts in the treatment of fractures of the radial head, the olecranon and the coronoid. Instr Course Lect 1995; 44: 175-185

5 Müller M, Nazarian S, Koch P. The AO Classification of long Bones. Berlin, Heidelberg, New York: Springer; 1987

${ }^{6}$ Nauth A, McKee MD, Ristevski B et al. Distal humeral fractures in adults. J Bone Joint Surg [Am] 2011; 93: 686-700

7 Papaioannou N, Babis GC, Kalavritinos J, Pantazopoulos $T$. Operative treatment of type $C$ intra-articular fractures of the distal humerus: the role of stability achieved at surgery on final outcome. Injury 1995; 26: 169-173

8 Proust J, Oksman A, Charissoux JL et al. Intraarticular fracture of the distal humerus: outcome after osteosynthesis in patients over 60 Rev Chir Orthop Reparatrice Appar Mot 2007; 93: 798-806

${ }^{9}$ Reising K, Hauschild O, Strohm PC, Suedkamp NP. Stabilisation of articular fractures of the distal humerus: early experience with a novel perpendicular plate system. Injury 2009; 40: 611-617
${ }^{10}$ Ring D, Jupiter JB. Fracture-dislocation of the elbow. J Bone Joint Surg [Am] 1998; 80: 566580

11 Schmidt-Horlohe $K$, Bonk A, Wilde $P$ et al. [Functional results after osteosynthesis of the distal humerus fracture with an anatomically precontoured, angular-stable double plate system]. Z Orthop Unfall 2010; 148: 300-308

12 Schmidt-Horlohe $K$, Siebenlist S, Stockle $U$, Hoffmann R. [Distal humeri fractures]. Z Orthop Unfall 2011; 149: 95-108

13 Schmidt-Horlohe K, Wilde P, Bonk A et al. Onethird tubular-hook-plate osteosynthesis for olecranon osteotomies in distal humerus type- $C$ fractures: a preliminary report of results and complications. Injury 2012; 43: 295-300

14 Siebenlist S, Stöckle U, Lucke M. Problematik osteoporotischer Frakturen am Ellenbogen. Obere Extremität 2009; 4: 160-167

15 Sodergard J, Sandelin J, Bostman O. Mechanical failures of internal fixation in $\mathrm{T}$ and $\mathrm{Y}$ fractures of the distal humerus. J Trauma 1992; 33: 687-690

16 Wright JG, Einhorn TA, Heckman JD. Grades of recommendation. J Bone Joint Surg [Am] 2005; 87: 1909-1910
Dr. med. Kay H. Schmidt-Horlohé

Assistenzarzt

Dr. med. Lars Becker

Assistenzarzt

Prof. Dr. med. Reinhard Hoffmann

Ärztlicher Direktor, Chefarzt

Abteilung für Unfallchirurgie und

Orthopädische Chirurgie

Berufsgenossenschaftliche Unfallklinik

Frankfurt am Main

Friedberger Landstraße 430

60389 Frankfurt am Main

kay.schmidt-horlohe@bgu-frankfurt.de 